\title{
A single-arm, prospective study of apatinib mesylate plus pemetrexed in patients of advanced non-squamous non-small cell lung cancer after failure of previous chemotherapy
}

\author{
Shuang Dong"^, Wuling Oü, Yi Zhong, Xianmin Zhu, Qian Cai, Jing Zhang, Fengming Ran, Yu Qian, \\ Jun Wang, Sheng Hu \\ Department of Medical Oncology, Hubei Cancer Hospital, Tongji Medical College, Huazhong University of Science and Technology, Wuhan, China \\ Contributions: (I) Conception and design: S Dong, W Ou, S Hu; (II) Administrative support: Y Zhong, X Zhu, Q Cai, J Zhang, F Ran, Y Qian, J \\ Wang; (III) Provision of study materials or patients: All authors; (IV) Collection and assembly of data: Y Zhong, X Zhu, Q Cai; (V) Data analysis and \\ interpretation: J Zhang, F Ran, Y Qian, J Wang; (VI) Manuscript writing: All authors; (VII) Final approval of manuscript: All authors. \\ \#These authors contributed equally to this work. \\ Correspondence to: Sheng Hu. Department of Medical Oncology, Hubei Cancer Hospital, Tongji Medical College, Huazhong University of Science \\ and Technology, Wuhan 430079, China. Email: ehusmn@163.com.
}

Background: The outcomes of advanced non-small cell lung cancer (NSCLC) patients after first- or second-line therapy are still discouraging due to a lack of effective treatment strategies. As a novel oral antiangiogenesis drug, apatinib, approved by the National Medical Products Administration of China only for advanced gastric cancer, has been increasingly used in off-label treatment across various cancer types in recent years, especially advanced NSCLC. It has shown strong anti-tumor efficacy and acceptable safety.

Methods: This prospective study (NCT02974933) was conducted in patients with advanced NSCLC, who had suffered disease progression from the first- or second-line treatment, in Hubei Cancer Hospital. Eligible patients were enrolled and administrated with apatinib mesylate $(500 \mathrm{mg}$ qd) in combination with pemetrexed $\left(500 \mathrm{mg} / \mathrm{m}^{2}\right.$, every 4 weeks). The primary endpoint was progression-free survival (PFS).

Results: From September 2016 to September 2019, a total of 21 advanced NSCLC patients were enrolled in Hubei Cancer Hospital. As of January 2021, treatment was discontinued in all patients, with 1 still in follow-up. There were 7/21 (33.3\%) patients who achieved objective response. The median PFS and median overall survival (OS) were 7.0 months (95\% CI: 6.15-7.85 months) and 13.0 months (95\% CI: $7.39-18.6$ months), respectively. Toxicities were tolerable or could be clinically managed. The most common grade 3-4 adverse events (AEs) were hypertension (14.3\%, 3/21), hand-foot syndrome $(4.7 \%, 1 / 21)$, and proteinuria $(4.7 \%, 1 / 21)$. Hematological toxicities were moderate, with rare occurrences of grade $3 / 4$ toxicities. During the period of treatment, there was no occurrence of treatment-related death.

Conclusions: Apatinib plus pemetrexed demonstrated promising efficacy and a high level of safety profile in previously heavily-treated NSCLC patients. More definitive studies on the combination of apatinib and pemetrexed are warranted

Keywords: Non-small cell lung cancer (NSCLC); apatinib; pemetrexed; efficacy; safety

Submitted Dec 22, 2021. Accepted for publication Jan 18, 2022.

doi: $10.21037 /$ atm-22-79

View this article at: https://dx.doi.org/10.21037/atm-22-79

$\wedge$ ORCID: 0000-0002-3627-9344. 


\section{Introduction}

Lung cancer ranks as one of the most common malignancies worldwide. In 2019 , approximately 228,000 people in the United States were diagnosed with lung cancer and more than half of newly diagnosed patients had metastases at the time of diagnosis (1). Non-small cell lung cancer (NSCLC) is the most frequently diagnosed pathological type, while adenocarcinoma (60\%) and squamous cell carcinoma $(15 \%)$ are the most common histological subtypes (2). In recent years, targeted therapies have been shown to play a profound role in the management of NSCLC $(2,3)$. Precise targeted therapies has revolutioned the treatment paradigm of NSCLC by inhibitors of epidermal growth factor receptor (EGFR), anaplastic lymphoma kinase (ALK), ROS proto-oncogene receptor tyrosine kinase 1 (ROS1), BRAF V600, and neurotrophic receptor tyrosine kinase (NTRK). Nevertheless, fewer than $25 \%$ of NSCLC patients who are harbor actinonable specific molecular alterations can benefit from these targeted therapies. With the expanding repertoire of feasible therapies for advanced NSCLC, immunotherapy targeting programmed cell death protein 1 (PD-1)/programmed cell death ligand 1 (PD-L1) axis have emerged as feasible therapeutics to provide long-term, durable benefit. When administered alone, however, only a fewer number of NSCLC patients $(<20 \%)$ respond to immune checkpoint inhibitors (2-5). Therefore, it is urgent to explore more effective therapies for NSCLC patients who experience progressive disease (PD) after first-line or even second-line treatment.

In the carcinogenesis, progression, and metastasis of NSCLC, angiogenesis plays a pivotal role by activating the vascular endothelial growth factor (VEGF)/vascular endothelial growth factor receptor-2 (VEGFR-2) axis (6). The currently available anti-angiogenic agents include anti-VEGF or VEGFR-2 monoclonal antibodies such as bevacizumab or ramucirumab, respectively, and small molecule tyrosine kinase inhibitors (TKIs) targeting VEGFRs in first- or second-line treatment $(7,8)$. In thirdline treatment, anlotinib, another kind of small molecule anti-angiogenic inhibitor, was shown to significantly improve overall survival (OS) and progression-free survival (PFS) in advanced NSCLC (9). Despite of anti-tumor effect, the survival benefit of anti-angiogenic inhibitor monotherapy is only modest, especially for previously heavily treated patients, which already implies that combination therapy should be of great interests.

Apatinib, a novel small molecule TKI that selectively inhibits VEGFR2, prevents the proliferation, migration, and neovascularization of endothelial cells. Apatinib has been approved for the third-line or beyond treatment of advanced or metastatic gastric cancer recurrent from chemotherapy in China. At the same time, apatinib has shown potential anti-tumor activity in the treatment of several solid tumors, whatever in monotherapy or combination with other antitumor agents (10-15). Furthermore, apatinib has been demonstrated to benefit patients of pretreated advanced NSCLC with a median PFS of 4.0 months in a retrospective study (12). Whereas, apatinib monotherapy brought limited survival benefit in NSCLC patients. Therefore, this phase II, single-arm, prospective study was conducted to evaluate the efficacy and safety of apatinib combined with pemetrexed in non-squamous NSCLC patients who had progressed on prior chemotherapies.

We present the following article in accordance with the TREND reporting checklist (available at https://atm. amegroups.com/article/view/10.21037/atm-22-79/rc).

\section{Methods}

\section{Study design and participants}

From September 2016 to September 2019, patients with advanced pretreated NSCLC were enrolled in Hubei Cancer Hospital. Eligible patients were: (I) histopathologically confirmed as non-squamous NSCLC, who had been progressed from previous treatment with more than 1 cycle of chemotherapy; (II) $\geq 18$ years of age with an Eastern Cooperative Oncology Group (ECOG) performance status of 0-2; (III) with a life expectancy of more than 12 weeks; (IV) $\geq 1$ measurable tumor lesions assessed according to the Response Evaluation Criteria in Solid Tumors version 1.1 (RECIST v1.1). Additional inclusion criteria were as follows: (I) adequate bone marrow function (white blood cell count $\geq 3.5 \times 10^{3}$ cells per $\mu \mathrm{L}$, absolute neutrophil count $\geq 1.5 \times 10^{3}$ cells per $\mu \mathrm{L}$, platelet count $\geq 100 \times 10^{3}$ platelets per $\mu \mathrm{L}$, and hemoglobin concentration $\geq 9.0 \mathrm{~g} / \mathrm{dL}$ ); (II) adequate liver function (bilirubin $\leq 1.5 \times$ upper limit of normal, alanine transaminase, aspartate transaminase $\leq 2.5 \times$ upper limit of normal) and kidney function (creatinine clearance $\geq 50 \mathrm{~mL} / \mathrm{min}$ and proteinuria $<1.0 \mathrm{~g} / 24 \mathrm{~h}$ ). Eligible patients were enrolled regardless of a treatment history of central nervous system (CNS) metastases or asymptomatic CNS disease.

Patients were excluded if they had the following: (I) had been treated with apatinib; (II) newly diagnosed untreated 
and/or active brain/meningeal metastases; (III) uncontrolled hypertension; (IV) gone though major surgical procedures within 4 weeks when first treatment initiated, present with any non-healing wound, peptic ulcer, incompletely healed bone fracture, active gastrointestinal tract bleeding, or significant hemoptysis $(\geq 2.5 \mathrm{~mL})$. Further exclusion criteria included significant cardiac morbidity, a medical history of cerebrovascular accident or pulmonary embolism, untreated deep venous thrombosis, transient ischemic attack in the last 6 months, any other life-threatening diseases, or malignancies other than non-melanoma skin cancer diagnosed in last 5 years.

The study was conducted in accordance with the Declaration of Helsinki (as revised in 2013) and Good Clinical Practice guideline (16), and was approved by the Ethics Committee of Hubei Cancer Hospital (LLHBCH2016KY-006). Written informed consent was obtained from all the patients before the performance of any study-related procedures.

\section{Procedures}

Apatinib (500 mg qd) was given continuously in a 28-day cycle until the occurrence of PD, unacceptable toxicity, patient withdrawal, or death. Dose interruptions or reductions were considered when grade $\geq 3$ hematological toxicities, grade $\geq 2$ non-hematological toxicities, or clinical intolerable grade 2 adverse events (AEs) occurred at any time. Dose reductions of apatinib were permitted to reduce to $250 \mathrm{mg}$ qd. Dose re-escalation of apatinib was permitted when drug-related AEs were ameliorated. Pemetrexed $\left(500 \mathrm{mg} / \mathrm{m}^{2}\right.$ every 28 days) was administered until PD or unacceptable toxicity, patient withdrawal, or death occurred.

\section{Endpoints and assessments}

Computed tomography (CT) and magnetic resonance imaging (MRI) were used to assess tumor response by the investigator at baseline, at the end of the first cycle, and then every 2 cycles until PD or discontinuation of treatment. Once aggravation of clinical symptoms occurred, CT scanning would be considered ahead of schedule. Tumor response was evaluated per the RECIST v1.1.

The primary endpoint was PFS, which was defined as the time interval from the initiation of treatment to the identification of PD or all-cause death, whichever occurred first. Secondary endpoints included OS, objective response rate (ORR), disease control rate (DCR), and safety profile. OS was defined as the interval between the start of treatment to death due to any cause, while DCR as the percentage of patients who achieved a complete response (CR), partial response (PR), or stable disease (SD).

Survival was continuously monitored throughout the treatment and every 2 months after discontinuation. Safety evaluation was performed during the study, including vital signs, physical examinations, routine blood tests, serum biochemistry, routine urine examination, and 12-lead electrocardiograms, etc. Treatment-related adverse events (AEs) were assessed according to the National Cancer Institute's Common Terminology Criteria for Adverse Events version 5.0 (CTCAE v5.0).

\section{Statistical analysis}

Simon's two-stage optimal design was used to estimate the sample size in our single arm phase II clinical study, based on response rate and survival probability. The exact levels of errors for type I and type II were $10 \%$ and $20 \%$, respectively. Our hypothesis is that therapy is ineffective if ORR $\leq 25 \%$. Through calculation by the formula which is open for public use (https://yeonhee.shinyapps.io/METTshinyapp/), in the first stage, at least 4 patients (25\%) had responded from 13 patients is the required threshold and then additional 11 patients would be enrolled for therapeutic intervention in the second stage, suggesting a total sample size is 24 .

The full analysis set (FAS) included all intentionto treat (ITT) patients to analyze the efficacy and safety profiles except those without safety data. The baseline characteristics of the ITT patients were summarized. The PFS and OS were estimated by the Kaplan-Meier method, meanwhile, the corresponding two-sided $95 \%$ confidence intervals (CIs) were calculated via the BrookmeyerCrowley method. A two-sided $\mathrm{P}$ value $<0.05$ was considered statistically significant. All analyses were performed with SPSS v23.0 (SPSS Inc., USA). This study had been registered on ClinicalTrials.gov (NCT02974933).

\section{Results}

\section{Patient disposition and clinical characteristics}

Between September 2016 and September 2019, a total of 23 patients with NSCLC were screened in Hubei Cancer Hospital, and 21 eligible patients were enrolled in the study (Figure 1). Patients were excluded mainly due to symptomatic brain metastasis and poor survival time. Table 


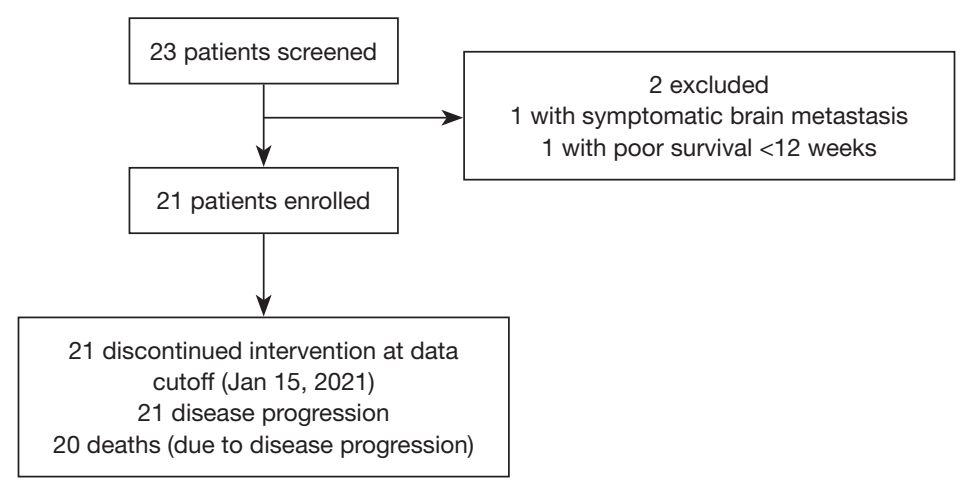

Figure 1 Flowchart of the patient screening and study processes.

Table 1 Patient demographics $(\mathrm{n}=21)$

\begin{tabular}{lc}
\hline Variables & Value \\
\hline Gender, $\mathrm{n}(\%)$ & $13(61.9)$ \\
Male & $8(38.1)$ \\
Female & $56.86 \pm 9.60$ \\
Age, median, years & $34.0-69.0$ \\
Min-max, years & \\
Smoking, $\mathrm{n}(\%)$ & $12(57.1)$ \\
Never & $9(42.9)$ \\
Given up & \\
EGFR, $\mathrm{n}$ (\%) & $9(42.9)$ \\
+ & $12(57.1)$ \\
- & \\
Treatment line, $\mathrm{n}(\%)$ & $2(9.5)$ \\
Second-line & $6(28.6)$ \\
Third-line & $16(76.2)$ \\
Fourth-line and above & \\
Brain metastases, $\mathrm{n}(\%)$ & \\
Yes & \\
\hline
\end{tabular}

EGFR, epidermal growth factor receptor.
1 shows the baseline characteristics of the 21 patients, with the median age of 57 years (range, 34-69 years), 13 (61.9\%) men, 12 (57.1\%) non-smokers, and 5 (23.8\%) brain metastases. All patients had received prior chemotherapy, of whom 13 patients $(61.9 \%)$ had received at least 4 prior lines of therapy, and 5 patients (23.8\%) had received other antiangiogenic therapies (Table 1).

\section{Efficacy}

All the 21 eligible patients were included in the analysis set. The median PFS and OS were 7.0 (95\% CI: 6.15-7.85) months (Figure 2) and 13.0 (95\% CI: 7.39-18.6) months (Figure 3), respectively. In detail, 7 (33.3\%) cases of PR, 12 (57.1\%) cases of SD, and $2(9.5 \%)$ cases of PD were achieved among these patients, without observation of CR (Table 2). Correspondingly, the ORR and DCR were $33.3 \%$ and $90.5 \%$, respectively. At the cut-off date, a total of 20 patients had died with 1 patient still alive, who were subsequently treated with apatinib combined with a PD-1 checkpoint inhibitor.

\section{Safety}

A total of 21 patients were included in the SAS, of whom 19/21 (90.5\%) patients experienced apatinib-related AEs. Based on the decision of the investigators, the AEs with clinical significance were summarized in Table 3. The most common grade $\geq 3$ AEs were hypertension (3/21, 14.3\%), 


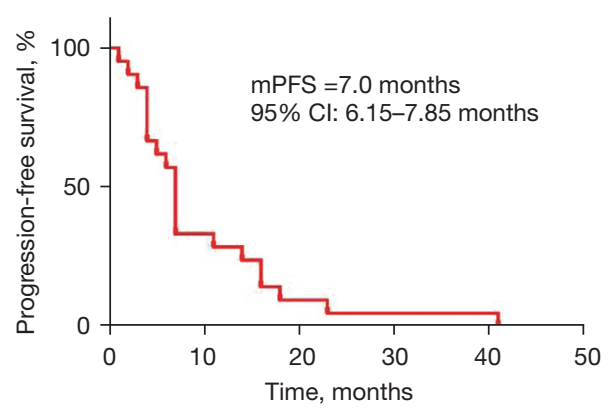

Figure 2 Kaplan-Meier analysis of PFS in patients treated with apatinib combined with pemetrexed $(n=21)$. The mPFS was 7.0 months (95\% CI: 6.15-7.85 months). PFS, progression-free survival; mPFS, median progression-free survival.

Table 2 Efficacy evaluation per RECIST v1.1

\begin{tabular}{lc}
\hline Efficacy & $\begin{array}{c}\text { Apatinib mesylate combined with } \\
\text { pemetrexed }(\mathrm{n}=21)\end{array}$ \\
\hline Objective response rate & $33.3 \%$ \\
Disease control rate & $90.5 \%$ \\
Complete response & 0 \\
Partial response & $7(33.3 \%)$ \\
Stable disease & $12(57.1 \%)$ \\
Disease progression & $2(9.5 \%)$ \\
\hline
\end{tabular}

leukopenia (2/21, 9.5\%), hand-foot syndrome (1/21, 4.7\%), proteinuria $(1 / 21,4.7 \%)$, and thrombocytopenia $(1 / 21$, $4.7 \%)$. In addition, 1 patient needed hospitalization due to grade 4 neutropenia. No grade 5 AEs, unexpected AEs, or treatment-related deaths were observed until the end of the study.

Dosage reduction of apatinib was reported in $11 / 21$ $(52.4 \%)$ patients during treatment, which was attributed to hand-foot syndrome $(6 / 21,28.6 \%)$, proteinuria $(4 / 21$, $19.0 \%)$, hypertension $(2 / 21,9.5 \%)$, leukopenia $(2 / 21,9.5 \%)$, mucositis (1/21, 4.7\%), and thrombocytopenia (1/21, 4.7\%).

\section{Discussion}

In recent years, novel therapies have been developed which have significantly improved the therapeutic effect of advanced NSCLC treatment (17-19). Chemotherapy is still a crucial option, especially for patients with no gene-sensitive mutations, those who experienced failure of targeted treatment, or those not suitable for

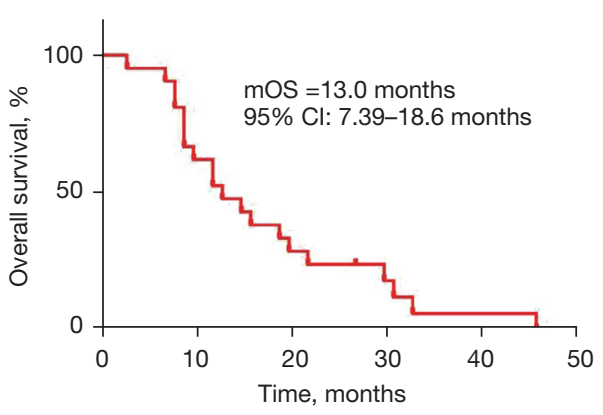

Figure 3 Kaplan-Meier analysis of OS in patients treated with apatinib combined with pemetrexed $(n=21)$. The mOS was 13.0 months (95\% CI: 7.39-18.6 months). OS, overall survival; mOS, median overall survival.

immunotherapy, although the prognosis is disappointing as most patients eventually relapse (20). Furthermore, effective treatment options are in urgent need for patients who have failed from previous treatment.

The growth of tumor cells is promoted by tumor angiogenesis supplying oxygen and nutrients, in which neovascularization, stimulated by activation of VEGF signaling, plays a vital role $(21,22)$. VEGFR2 is a major member of the VEGFR family, mainly including VEGFR1, VEGFR2, VEGFR3, and is considered as the most important receptor impacting on tumor angiogenesis. A meta-analysis showed that the addition of anti-angiogenic TKIs to chemotherapy significantly improved ORR and median PFS when compared with chemotherapy alone for advanced NSCLC (23). Pemetrexed, belonging to the class of antifolate-metabolizing chemicals that inhibit the synthesis of DNA and RNA, is an important recommended drug for non-squamous NSCLC (24). With fewer side effects, pemetrexed may be an optional drug for patient treated by multiline treatment. Additional, anti-angiogenic TKIs plus pemetrexed may lead to better compliance and good tolerability.

Apatinib, a small molecule VEGFR TKI targeting VEGFR2 and other receptor tyrosine kinases, such as c-kit, RET, and c-src, is a first generation oral antiangiogenesis agent developed in China (25). The efficacy of apatinib monotherapy has been demonstrated in various cancer types, such as gastric cancer (26), liver cancer (27), lung cancer $(28,29)$, and thyroid cancer $(30)$. Our study demonstrated that low dose apatinib combined with pemetrexed could provide beneficial clinical outcomes and a favorable safety profile in advanced NSCLC patients. In this study, apatinib combined with pemetrexed produced 
Table 3 Adverse events

\begin{tabular}{|c|c|c|c|c|c|c|}
\hline Adverse events & \multicolumn{5}{|c|}{ Grade $(n=21)$} & Incidence (\%) \\
\hline Hypertension & 3 & 3 & 0 & 0 & 6 & 28.6 \\
\hline Proteinuria & 12 & 1 & 0 & 0 & 13 & 61.9 \\
\hline Hand-foot syndrome & 9 & 1 & 0 & 0 & 10 & 47.6 \\
\hline Leukopenia & 7 & 0 & 2 & 0 & 9 & 42.9 \\
\hline Anemia & 12 & 1 & 0 & 0 & 13 & 61.9 \\
\hline Thrombocytopenia & 5 & 1 & 0 & 0 & 6 & 28.6 \\
\hline Transaminase elevation & 7 & 0 & 0 & 0 & 7 & 33.3 \\
\hline
\end{tabular}

a median PFS of 7.0 months, while the median PFS of apatinib monotherapy in patients with advanced nonsquamous NSCLC was 2.93 months in a phase II trial (14), indicating that apatinib combined with pemetrexed could provide effective clinical outcomes after failure of previous chemotherapy. Furthermore, apatinib monotherapy (250 to $750 \mathrm{mg} \mathrm{qd}$ ) produced a median PFS and median OS of 3 (95\% CI: 2.6-3.4) and 7.4 (95\% CI: 1.3-13.5) months, respectively, in advanced non-squamous NSCLC after multi-line treatments (29). In contrast,), it showed a longer survival in this study, with a median OS of 13.0 months. Apatinib reverses ATP binding cassette subfamily B member 1 (ABCB1; P-glycoprotein, P-gp)- and ATP-binding cassette transporter G2 (ABCG2; breast cancer resistance protein, $\mathrm{BCRP}$ )-mediated multidrug resistance (MDR) by directly inhibiting the function of ABCB1 and ABCG2, elevating the intracellular concentrations of the chemotherapeutic substrate (31). These findings could reveal the potential mechanism of better anti-tumor effect of combined therapy.

It is of particular concern that there were 5 patients who had progressed on other anti-angiogenic treatments of bevacizumab or endostatin, still achieving disease control in this study, suggesting that the anti-tumor effect of apatinib was significantly different. Consequently, apatinib could provide a potential opportunity for patients who had failed in previous anti-angiogenic therapy. Moreover, brain lesions decreased in 5 patients with asymptomatic brain metastases, suggesting that apatinib could be an option for NSCLC patients with asymptomatic brain metastases.

The AE profiles of apatinib combined with pemetrexed were manageable in the treatment of advanced NSCLC patients. The most frequent apatinib-related AEs were hypertension, hand-foot syndrome, proteinuria, and fatigue. Most AEs were mild and manageable. Only a few patients experienced grade 3 or 4 toxicity or serious AEs, and no irreversible toxicity occurred. The incidence of these AEs was even lower than that caused by a high dose of apatinib monotherapy (29). The majority of adverse effects were reversible and blood pressure returned to normal after stopping discontinuation of apatinib.

It was found that the level of tumor phosphorylated VEGFR2 (p-VEGFR2) and hypertension were independent predictive factors for the prognosis of patients and are considered as potential predictors of apatinib treatment (32). Nevertheless, this study did not find a correlation between hypertension and efficacy, which may be attributed to the small sample size.

In conclusion, apatinib combined with pemetrexed is promising in the treatment of patients with advanced NSCLC who failed on standard treatment, with manageable toxicities. Apatinib plus pemetrexed may be a new treatment option for patients who have failed previous chemotherapy, EGFR-TKI treatment, or anti-angiogenic treatment.

As a single-arm study, there were some inevitable limitations. There may exist some selection bias as no control group. The small sample size and high rate of dosage adjustment may play a part. Despite these limitations, this study provided evidence for apatinib plus chemotherapy or novel anti-tumor agents, such as immunotherapy, in patients with advanced NSCLC. Therefore, further large scale clinical trials are urgently necessary in the future. 


\section{Acknowledgments}

The authors sincerely thank all patients who participated in the study.

Funding: None.

\section{Footnote}

Reporting Checklist: The authors have completed the TREND reporting checklist. Available at https://atm. amegroups.com/article/view/10.21037/atm-22-79/rc

Data Sharing Statement: Available at https://atm.amegroups. com/article/view/10.21037/atm-22-79/dss

Conflicts of Interest: All authors have completed the ICMJE uniform disclosure form (available at https://atm. amegroups.com/article/view/10.21037/atm-22-79/coif). The authors have no conflicts of interest to declare.

Ethical Statement: The authors are accountable for all aspects of the work in ensuring that questions related to the accuracy or integrity of any part of the work are appropriately investigated and resolved. The study was conducted in accordance with the Declaration of Helsinki (as revised in 2013), and was approved by the Ethics Committee of Hubei Cancer Hospital (LLHBCH2016KY-006). Written informed consent was obtained from all the patients before the performance of any study-related procedures.

Open Access Statement: This is an Open Access article distributed in accordance with the Creative Commons Attribution-NonCommercial-NoDerivs 4.0 International License (CC BY-NC-ND 4.0), which permits the noncommercial replication and distribution of the article with the strict proviso that no changes or edits are made and the original work is properly cited (including links to both the formal publication through the relevant DOI and the license). See: https://creativecommons.org/licenses/by-nc-nd/4.0/.

\section{References}

1. Miller KD, Nogueira L, Mariotto AB, et al. Cancer treatment and survivorship statistics, 2019. CA Cancer J Clin 2019;69:363-85.

2. NCCN Clinical Practice Guidelines in Oncology (NCCN Guidelines). Non-small cell lung cancer. National Comprehensive Cancer, Network 2020. V2; 2019.
3. Hirsch FR, Scagliotti GV, Mulshine JL, et al. Lung cancer: current therapies and new targeted treatments. Lancet 2017;389:299-311.

4. Ricciuti B, Brambilla M, Metro G, et al. Targeting NTRK fusion in non-small cell lung cancer: rationale and clinical evidence. Med Oncol 2017;34:105.

5. Reck M. Pembrolizumab as first-line therapy for metastatic non-small-cell lung cancer. Immunotherapy 2018;10:93-105.

6. Goel HL, Mercurio AM. VEGF targets the tumour cell. Nat Rev Cancer 2013;13:871-82.

7. Sandler A, Gray R, Perry MC, et al. Paclitaxel-carboplatin alone or with bevacizumab for non-small-cell lung cancer. N Engl J Med 2006;355:2542-50. Erratum in: N Engl J Med 2007;356:318.

8. Garon EB, Ciuleanu TE, Arrieta O, et al. Ramucirumab plus docetaxel versus placebo plus docetaxel for secondline treatment of stage IV non-small-cell lung cancer after disease progression on platinum-based therapy (REVEL): a multicentre, double-blind, randomised phase 3 trial. Lancet 2014;384:665-73.

9. Han B, Li K, Wang Q, et al. Effect of Anlotinib as a Third-Line or Further Treatment on Overall Survival of Patients With Advanced Non-Small Cell Lung Cancer: The ALTER 0303 Phase 3 Randomized Clinical Trial. JAMA Oncol 2018;4:1569-75. Erratum in: JAMA Oncol 2018;4:1625.

10. Li J, Qin S, Xu J, et al. Randomized, Double-Blind, Placebo-Controlled Phase III Trial of Apatinib in Patients With Chemotherapy-Refractory Advanced or Metastatic Adenocarcinoma of the Stomach or Gastroesophageal Junction. J Clin Oncol 2016;34:1448-54.

11. Tian S, Quan H, Xie C, et al. YN968D1 is a novel and selective inhibitor of vascular endothelial growth factor receptor-2 tyrosine kinase with potent activity in vitro and in vivo. Cancer Sci 2011;102:1374-80.

12. Liu Z, Ou W, Li N, et al. Apatinib monotherapy for advanced non-small cell lung cancer after the failure of chemotherapy or other targeted therapy. Thorac Cancer 2018;9:1285-90.

13. Chen Y, Gong J, Zhou H, et al. Apatinib for EGFR-TKI and chemotherapy refractory in an advanced lung cancer patient: a case report. J Thorac Dis 2018;10:E564-9.

14. Zhang D, Zhang C, Huang J, et al. Clinical investigation of the efficacy and toxicity of apatinib (YN968D1) in stage III/IV non-small cell lung cancer after second-line chemotherapy treatment: A retrospective study. Thorac Cancer 2018;9:1754-62.

15. Wu F, Zhang S, Xiong A, et al. A Phase II Clinical Trial of 
Apatinib in Pretreated Advanced Non-squamous Non-smallcell Lung Cancer. Clin Lung Cancer 2018;19:e831-42.

16. Chinese Good Clinical Practice Guideline. Available online: https://www.nmpa.gov.cn/xxgk/fgwj/ bmgzh/20030806010101443.html

17. Mok TS, Wu YL, Thongprasert S, et al. Gefitinib or carboplatin-paclitaxel in pulmonary adenocarcinoma. N Engl J Med 2009;361:947-57.

18. Zhou C, Wu YL, Chen G, et al. Erlotinib versus chemotherapy as first-line treatment for patients with advanced EGFR mutation-positive non-small-cell lung cancer (OPTIMAL, CTONG-0802): a multicentre, open-label, randomised, phase 3 study. Lancet Oncol 2011;12:735-42.

19. Hirsh V, Park K, Tan EH, et al. Afatinib vs gefitinib in patients (pts) with EGFR mutation-positive (EGFRm+) NSCLC: overall survival (OS) data from LUX-Lung 7 (LL7). European Journal of Cancer 2017;72:S184.

20. Zukin M, Barrios CH, Pereira JR, et al. Randomized phase III trial of single-agent pemetrexed versus carboplatin and pemetrexed in patients with advanced non-small-cell lung cancer and Eastern Cooperative Oncology Group performance status of 2. J Clin Oncol 2013;31:2849-53.

21. Ferrara N. VEGF and the quest for tumour angiogenesis factors. Nat Rev Cancer 2002;2:795-803.

22. Jayson GC, Kerbel R, Ellis LM, et al. Antiangiogenic therapy in oncology: current status and future directions. Lancet 2016;388:518-29.

23. Yu GC, Yang J, Ye B, et al. Apatinib in the treatment of advanced non-small-cell lung cancer: A meta-analysis. Math Biosci Eng 2019;16:7659-70.

24. Tomasini P, Barlesi F, Mascaux C, et al. Pemetrexed for advanced stage nonsquamous non-small cell lung cancer: latest evidence about its extended use and outcomes. Ther

Cite this article as: Dong $\mathrm{S}$, Ou W, Zhong $\mathrm{Y}$, Zhu X, Cai Q, Zhang J, Ran F, Qian Y, Wang J, Hu S. A singlearm, prospective study of apatinib mesylate plus pemetrexed in patients of advanced non-squamous non-small cell lung cancer after failure of previous chemotherapy. Ann Transl Med 2022;10(2):101. doi: 10.21037/atm-22-79
Adv Med Oncol 2016;8:198-208.

25. Scott LJ. Apatinib: A Review in Advanced Gastric Cancer and Other Advanced Cancers. Drugs 2018;78:747-58.

26. Li J, Qin S, Xu J, et al. Apatinib for chemotherapyrefractory advanced metastatic gastric cancer: results from a randomized, placebo-controlled, parallel-arm, phase II trial. J Clin Oncol 2013;31:3219-25.

27. Liang Q, Kong L, Du Y, et al. Antitumorigenic and antiangiogenic efficacy of apatinib in liver cancer evaluated by multimodality molecular imaging. Exp Mol Med 2019;51:1-11.

28. Xu Y, Huang Z, Lu H, et al. Apatinib in patients with extensive-stage small-cell lung cancer after second-line or third-line chemotherapy: a phase II, single-arm, multicentre, prospective study. Br J Cancer 2019;121:640-6.

29. Wu D, Liang L, Nie L, et al. Efficacy, safety and predictive indicators of apatinib after multilines treatment in advanced nonsquamous nonsmall cell lung cancer: Apatinib treatment in nonsquamous NSCLC. Asia Pac J Clin Oncol 2018;14:446-52.

30. Chen K, Gao Y, Shi F, et al. Apatinib-treated advanced medullary thyroid carcinoma: a case report. Onco Targets Ther 2018;11:459-63.

31. Mi YJ, Liang YJ, Huang HB, et al. Apatinib (YN968D1) reverses multidrug resistance by inhibiting the efflux function of multiple ATP-binding cassette transporters. Cancer Res 2010;70:7981-91.

32. Fan M, Zhang J, Wang Z, et al. Phosphorylated VEGFR2 and hypertension: potential biomarkers to indicate VEGFdependency of advanced breast cancer in anti-angiogenic therapy. Breast Cancer Res Treat 2014;143:141-51.

(English Language Editor: C. Betlazar-Maseh) 